PROCEEDINGS OF THE

AMERICAN MATHEMATICAL SOCIETY

Volume 126, Number 2, February 1998, Pages 561-567

S 0002-9939(98)04197-5

\title{
ROTATION INVARIANT AMBIGUITY FUNCTIONS
}

\author{
QINGTANG JIANG
}

(Communicated by J. Marshall Ash)

\begin{abstract}
Let $W(\psi ; x, y)$ be the wideband ambiguity function. It is obtained in this note that $y^{-\frac{\alpha+2}{2}} W(\psi ; x, y)(\alpha>-1)$ is $S O(2)$-invariant if and only if the Fourier transform of $\psi$ is a Laguerre function.
\end{abstract}

\section{INTRODUCTION}

For $g \in L^{2}(\mathbb{R})$, the continuous Gabor transform (or windowed Fourier transform) of $f \in L^{2}(\mathbb{R})$ with analyzing function $g$ is defined by

$$
\Psi_{g} f(x, y):=\int_{-\infty}^{+\infty} f(t) e^{-2 \pi i y t} \overline{g(t-x)} d t .
$$

It was introduced by Gabor for study of communication theory ([4]). In (1.1), $x$ is the time variable and $y$ is the frequency variable. The transform $\Psi_{g} f(x, y)$ of $f$ is formed by shifting the window function $g$ so that it is centered at $x$, then taking the Fourier transform. In this way, $\Psi_{g} f(x, y)$ displays the frequency content of $f$ near time $x$. For $f \in L^{2}(\mathbb{R})$, it can be reconstructed from $\Psi_{g} f(x, y)$ :

$$
f(t)=\frac{1}{\|g\|_{2}^{2}} \int_{\mathbb{R}^{2}} \Psi_{g} f(x, y) g(t-x) e^{2 \pi i y t} d x d y .
$$

For $f, g \in L^{2}(\mathbb{R})$, the radar cross-ambiguity function of $f, g$ is defined to be

$$
H(f, g ; x, y):=\int_{-\infty}^{+\infty} f\left(t+\frac{1}{2} x\right) \overline{g\left(t-\frac{1}{2} x\right)} e^{-2 \pi i y t} d t .
$$

From (1.1), (1.2), one knows $\Psi_{g} f(x, y)$ is exactly $H(f, g ; x, y)$ except for a phase factor. Both $\Psi_{g} f(x, y)$ and $H(f, g ; x, y)$ are related to the representation of the Weyl-Heisenberg group ( $[15],[12])$. For $f \in L^{2}(\mathbb{R})$, denote $H(f ; x, y):=$ $H(f, f ; x, y)$. Function $H(f ; x, y)$ is called the radar auto-ambiguity function or narrowband ambiguity function with respect to signal $f$. Ambiguity functions play an important role in radar analysis and design since they were introduced by Woodward (see [20], [19], [15]). Properties of $H(f, f ; x, y), H(f ; x, y)$ and their applications can be found in many literatures, e.g. [19], [15]. One of these properties is that $H(f ; x, y)$, as a function on $\mathbb{R}^{2}$, is $S O(2)$-invariant (or rotation-invariant) if and only if $f(x)$ is a Hermite function, i.e. there exists a nonnegative integer $m$

Received by the editors October 25, 1995 and, in revised form, August 23, 1996.

1991 Mathematics Subject Classification. Primary 42C05, 42C99.

Key words and phrases. Ambiguity function, rotation invariant, Laguerre function.

(C)1998 American Mathematical Society 
such that $f(x)=c h_{m}(x) e^{-x^{2}}$, here $h_{m}(x)$ is the Hermite polynomial of degree $m$, see [19], [15].

Originally proposed as an alternative to windowed Fourier transform, wavelet transform has its applications in many fields (see [6], [2]). Let $H^{2}(\mathbb{R})$ denote the Hardy space, the subspace of $L^{2}(\mathbb{R})$ consisting of functions $\psi$ with supp $\widehat{\psi} \subset[0,+\infty)$. The continuous wavelet transform of $f \in H^{2}(\mathbb{R})$ with analyzing function $\psi \in$ $H^{2}(\mathbb{R})$, denoted by $W_{\psi}$, is defined by

$$
W_{\psi} f(x, y):=\frac{1}{\sqrt{y}} \int_{-\infty}^{+\infty} f(t) \overline{\psi\left(\frac{t-x}{y}\right)} d t .
$$

Continuous wavelet transform is associated to the square integrable representation of the affine group " $a x+b$ " (see [5]). When $\psi$ satisfies the following condition

$$
C_{\psi}:=2 \pi \int_{0}^{+\infty}|\widehat{\psi}(\omega)|^{2} \frac{d \omega}{\omega}<+\infty
$$

then $f(x)$ can be reconstructed from $W_{\psi} f(x, y)$ as from $\Psi_{g} f(x, y)$. In this case

$$
f(x)=\frac{1}{C_{\psi}} \int_{0}^{+\infty} \int_{\mathbb{R}} W_{\psi} f(b, a) \frac{1}{\sqrt{a}} \psi\left(\frac{x-b}{a}\right) \frac{d a d b}{a^{2}} .
$$

Equation (1.5) holds at least "in the weak sense", i.e. taking inner product of both sides of (1.5) with any $g \in H^{2}(\mathbb{R})$ and commuting the inner product with the integral over $a, b$ in the right-hand side leads to a true formula, which in fact is the Moyal formula. The convergence of the integral in (1.5) also holds in the following "strong sense" (see [2]):

$$
\lim _{\delta \rightarrow 0, A, B \rightarrow+\infty}\left\|f(x)-C_{\psi}^{-1} \int_{\delta<a<A} \int_{|b|<B} W_{\psi} f(b, a) \frac{1}{\sqrt{a}} \psi\left(\frac{x-b}{a}\right) \frac{d a d b}{a^{2}}\right\|_{2}=0 .
$$

For $\psi, f \in H^{2}(\mathbb{R})$, let $W(\psi, f ; x, y):=W_{\psi} f(x, y)$ be the wideband crossambiguity function of $\psi, f$ and $W(\psi ; x, y):=W(\psi, \psi ; x, y)$ the wideband ambiguity function. Such ambiguity functions were studied by Swick in [16](1967), [17](1969). The renewed interest in the wideband functions ([1] , [12], [10], [14], [18], [21], [8]) seems to have been inspired by the development of wavelet analysis. In this note, we will consider the $S O(2)$-invariant properties of $W(\psi ; x, y)$. The rotation invariance of wideband ambiguity functions would be of interest for applications in radar/sonar analysis or design. In the following, when considering the $S O(2)$-invariant properties of ambiguity functions, we will assume that $\psi \in H^{2}(\mathbb{R})$, $\widehat{\psi}$ is real and $\psi(x)$ having some smooth and decaying properties at infinity which insure that $\widehat{\psi}^{\prime \prime}(\omega)$ exists on $\mathbb{R}_{+}^{*}:=(0,+\infty)$ and $\widehat{\psi}(\omega) \widehat{\psi}^{\prime}(\omega), \omega \widehat{\psi}(\omega) \widehat{\psi}^{\prime \prime}(\omega) \in L^{1}\left(\mathbb{R}_{+}^{*}\right)$. Let $\mathcal{A}$ denote the set of all such functions.

\section{MAIN RESULTS}

For $\psi \in \mathcal{A}$, let $W(\psi ; x, y)$ be the wideband ambiguity function of $\psi$ defined as above. As a function on the upper half plane $U$, if $W(\psi ; x, y)$ is called $S O(2)$ invariant when it satisfies

$$
W\left(\psi ; x_{\theta}, y_{\theta}\right)=W(\psi ; x, y), \text { with } x_{\theta}+i y_{\theta}=\frac{(x+i y) \cos \frac{\theta}{2}-\sin \frac{\theta}{2}}{(x+i y) \sin \frac{\theta}{2}+\cos \frac{\theta}{2}},
$$

then we can get as below that there is no $S O(2)$-invariant $\psi \in \mathcal{A}$. In the following we will consider the ambiguity functions with a different dilation factor. 
For $\alpha>-1$, let $L^{\alpha 2}(U)$ denote the function space consisting of functions on the upper half plane $U$ square integrable with measure $y^{\alpha} d x d y$. For $\psi \in H^{2}(\mathbb{R})$, define the wavelet transform of $f \in H^{2}$ with a different dilation by

$$
W^{\alpha}(\psi, f ; x, y)=W_{\psi}^{\alpha} f(x, y):=\frac{1}{y^{\nu}} W_{\psi} f(x, y),
$$

where

$$
\nu:=\alpha+2
$$

and $h=\frac{1}{\nu}$ is the Planck constant in the terminology of quantum mechanics. If $\psi$ satisfies $(1.4), W_{\psi}^{\alpha}$ is an isometry (up to a constant) from $H^{2}(\mathbb{R})$ into $L^{\alpha 2}(U)$. We will consider the rotation invariant properties of the ambiguity function

$$
W^{\alpha}(\psi ; x, y):=W^{\alpha}(\psi, \psi ; x, y)=\frac{1}{y^{\nu+\frac{1}{2}}} \int_{-\infty}^{+\infty} \psi(t) \overline{\psi\left(\frac{t-x}{y}\right)} d t .
$$

Let $S L(2, \mathbb{R})$ denote the special linear group. For $g \in S L(2, \mathbb{R})$, it acts on $U$ via the transformations

$$
g: z \rightarrow g z:=g(z)=\frac{a z+b}{c z+d}, \quad \text { with } \quad g^{-1}=\left(\begin{array}{ll}
a & b \\
c & d
\end{array}\right),
$$

and it induces the action on $L^{\alpha 2}(U)$ via

$$
U_{g}^{\nu}: F(z) \rightarrow F(g z)\left\{g^{\prime}(z)\right\}^{\frac{\nu}{2}}=F(g z)(c z+d)^{-\nu} .
$$

Let $S O(2)$ be the special rotation group, the maximal compact subgroup of $S L(2, \mathbb{R})$, then elements $g \in S O(2)$ are given by

$$
g=\left(\begin{array}{cc}
\cos \frac{\theta}{2} & \sin \frac{\theta}{2} \\
-\sin \frac{\theta}{2} & \cos \frac{\theta}{2}
\end{array}\right), \text { with } g^{-1}=\left(\begin{array}{rr}
\cos \frac{\theta}{2} & -\sin \frac{\theta}{2} \\
\sin \frac{\theta}{2} & \cos \frac{\theta}{2}
\end{array}\right), 0 \leq \theta<2 \pi .
$$

Let $R_{\theta}$ be the restriction of $U_{g}^{\nu}$ to $S O(2)$ given by

$$
R_{\theta} F(z):=\frac{\left(i \sin \frac{\theta}{2}+\cos \frac{\theta}{2}\right)^{\nu}}{\left(z \sin \frac{\theta}{2}+\cos \frac{\theta}{2}\right)^{\nu}} F\left(\frac{z \cos \frac{\theta}{2}-\sin \frac{\theta}{2}}{z \sin \frac{\theta}{2}+\cos \frac{\theta}{2}}\right),
$$

where $c_{\theta}:=\left(i \sin \frac{\theta}{2}+\cos \frac{\theta}{2}\right)^{\nu}$. Adding the constant $c_{\theta}$ in the definition of $R_{\theta}$ is to assure that $R_{\theta} F(i)=F(i)$. In fact the point $i$ is the rotation center. If $R_{\theta} F(z)=F(z)$, then $F(z)$ is called $S O(2)$-invariant.

Let $\mathbb{Z}_{+}$denote the set of all nonnegative integers and in this note we would consider the problem in the case $\alpha \in \mathbb{Z}_{+}$. We have

Theorem 2.1. For $\alpha \in \mathbb{Z}_{+}$and $\psi \in \mathcal{A}$, let $W^{\alpha}(\psi ; x, y)$ be the ambiguity function of $\psi$ defined by $(2.1)$, then $W^{\alpha}(\psi ; x, y)$ is $S O(2)$-invariant if and only if there exists $k \in \mathbb{Z}_{+}, k<\frac{\alpha+1}{2}$, such that

$$
\widehat{\psi}(\omega)= \begin{cases}c(2 \omega)^{\frac{\alpha+1}{2}-k} L_{k}^{(\alpha+1-2 k)}(2 \omega) e^{-\omega}, & \text { for } \omega \geq 0, \\ 0, & \text { for } \omega<0\end{cases}
$$

where $c$ is a nonzero constant and $L_{k}^{(\alpha)}(\omega)$ is the Laguerre polynomial of degree $k$.

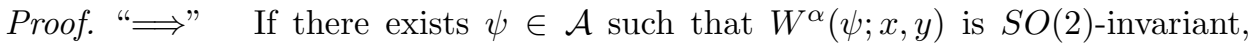
then $R_{\theta} W^{\alpha}(\psi ; x, y)=W^{\alpha}(\psi ; x, y)$ for all $\theta$ and hence

$$
\left.\frac{d\left(R_{\theta} W^{\alpha}(\psi ; x, y)\right)}{d \theta}\right|_{\theta=0}=0 .
$$


For appropriate functions $F(z)$ on $U$, it follows by a direct calculation

$$
\left.\frac{d R_{\theta} F(z)}{d \theta}\right|_{\theta=0}=\frac{i \nu}{2} F(z)-\frac{\nu}{2} z F(z)+\frac{y^{2}-x^{2}-1}{2} \frac{\partial F(x, y)}{\partial x}-x y \frac{\partial F(x, y)}{\partial y} .
$$

From the definition of $W_{\psi}^{\alpha} f(x, y)$,

$$
W_{\psi}^{\alpha} f(x, y)=\frac{y^{-\frac{\nu-1}{2}}}{2 \pi} \int_{0}^{+\infty} \widehat{\psi}(y \omega) \widehat{f}(\omega) e^{i \omega x} d \omega .
$$

Therefore we would consider functions with the form of

$$
F(z)=y^{-\frac{\nu-1}{2}} \int_{0}^{+\infty} h(y \omega) g(\omega) e^{i \omega x} d \omega
$$

where $z=x+i y, h(\omega)$ and $g(\omega)$ are real functions on $\mathbb{R}_{+}^{*}$ with $h^{\prime \prime}(\omega), g^{\prime \prime}(\omega)$ existing and having some decay properties at infinity. For such kind function $F(z)$,

$$
\begin{aligned}
- & \left.2 i \frac{d R_{\theta} F(z)}{d \theta}\right|_{\theta=0} \\
= & \nu(1-y) F(z)+i x F(z)+\left(y^{2}-x^{2}-1\right) y^{-\frac{\nu-1}{2}} \int_{0}^{+\infty} h(y \omega) \omega g(\omega) e^{i \omega x} d \omega \\
& +2 i x y y^{-\frac{\nu-1}{2}} \int_{0}^{+\infty} h^{\prime}(y \omega) \omega g(\omega) e^{i \omega x} d \omega .
\end{aligned}
$$

Let $D_{\nu}$ denote the differential operator of functions on $\mathbb{R}_{+}^{*}$ defined by

$$
D_{\nu}:=-\omega^{2} \frac{d^{2}}{d \omega^{2}}-\omega \frac{d}{d \omega}+\omega^{2}-\nu \omega+\frac{(\nu-1)^{2}}{4} .
$$

Then by a direct calculation and (2.5), one has

$$
\begin{aligned}
\int_{0}^{+\infty} & h(y \omega) \frac{1}{\omega} D_{\nu} g(\omega) e^{i \omega x} d \omega-\int_{0}^{+\infty} g(\omega) \frac{1}{\omega} D_{\nu} h(y \omega) e^{i \omega x} d \omega \\
= & -\left.2 i y^{\frac{\nu-1}{2}} \frac{d R_{\theta} F(z)}{d \theta}\right|_{\theta=0} .
\end{aligned}
$$

Let $F(z)=W^{\alpha}(\psi ; x, y)$; then $(2.4)$ and $(2.7)$ lead to

$$
\int_{0}^{\infty} \widehat{\psi}(y \omega) \frac{1}{\omega} D_{\nu} \widehat{\psi}(\omega) e^{i \omega x} d \omega=\int_{0}^{\infty} \widehat{\psi}(\omega) \frac{1}{\omega} D_{\nu} \widehat{\psi}(y \omega) e^{i \omega x} d \omega
$$

and hence $\widehat{\psi}(y \omega) D_{\nu} \widehat{\psi}(\omega)=\widehat{\psi}(\omega) D_{\nu} \widehat{\psi}(y \omega)$ for all $y, \omega \in R_{+}^{*}$. Therefore one can get that $\widehat{\psi}(\omega)$ is an eigenfunction of $D_{\nu}$. The differential operator $D_{\nu}$ has spectra (see $[3],[13])$ :

$$
\sigma\left(D_{\nu}\right)=\left\{\left(\frac{\nu-1}{2}\right)^{2}-\left(\frac{\nu-1}{2}-k\right)^{2}, k \in \mathbb{Z}_{+}, k<\frac{\nu-1}{2}\right\} \cup\left\{\left[\left(\frac{\nu-1}{2}\right)^{2},+\infty\right)\right\} .
$$

For $k<\frac{\nu-1}{2}$, denote $\lambda_{k}:=\left(\frac{\nu-1}{2}\right)^{2}-\left(\frac{\nu-1}{2}-k\right)^{2}$, and let $\widehat{\psi}_{k}(\omega)$ be the eigenfunction of $D_{\nu}$ corresponding to $\lambda_{k}$, i.e.

$$
D_{\nu} \widehat{\psi}_{k}(\omega)=\lambda_{k} \widehat{\psi}_{k}(\omega)
$$

And let $\varphi$ be the function defined by $\widehat{\psi}_{k}(\omega)=(2 \omega)^{-\frac{1}{2}} \varphi(2 \omega)$. Then by (2.8),

$$
\varphi^{\prime \prime}(t)+\left(-\frac{1}{4}+\frac{\nu}{2 t}+\frac{1+4 \lambda_{k}-(\nu-1)^{2}}{4 t^{2}}\right) \varphi(t)=0 .
$$


Equation (2.9) is just the "Whittaker's differential equation" (see [11]) and it has solution $M_{\mathcal{N}, \mu_{k}}$, the Whittaker's function, given by

$$
M_{\mathcal{N}, \mu_{k}}(t)=e^{-\frac{t}{2}} t^{\mu_{k}+\frac{1}{2}}{ }_{1} F_{1}\left(\mu_{k}+\frac{1}{2}-\mathcal{N} ; 1+2 \mu_{k} ; t\right),
$$

where $\mathcal{N}=\frac{\nu}{2}, \mu_{k}=\frac{\nu-1}{2}-k$. Thus

$$
\begin{aligned}
\widehat{\psi}_{k}(\omega) & =(2 \omega)^{-\frac{1}{2}} M_{\mathcal{N}, \mu_{k}}(2 \omega)=(2 \omega)^{\frac{\nu-1-2 k}{2}} e^{-\omega}{ }_{1} F_{1}(-k ; \nu-1-2 k ; 2 \omega) \\
& =(2 \omega)^{\frac{\alpha+1-2 k}{2}} e^{-\omega} L_{k}^{(\alpha+1-2 k)}(2 \omega) .
\end{aligned}
$$

For the continuous spectra $\lambda$ of $D_{\nu}$, let $\widehat{\psi}_{\lambda}$ be the corresponding eigenfunction; then one can get as above that

$$
\widehat{\psi}_{\lambda}(\omega)=(2 \omega)^{\mu_{\lambda}} e^{-\omega}{ }_{1} F_{1}\left(\mu_{\lambda}+\frac{1}{2}-\frac{\nu}{2} ; 1+2 \mu_{\lambda} ; 2 \omega\right),
$$

where $\mu_{\lambda}= \pm i \sqrt{\lambda-\left(\frac{\nu-1}{2}\right)^{2}}$. Such $\psi_{\lambda}$ is not in $\mathcal{A}$ since $\widehat{\psi}_{\lambda}(\omega)$ is not a real function.

"£" For any $k \in \mathbb{Z}_{+}, k<\frac{\alpha+1}{2}$, let $\psi_{k} \in \mathcal{A}$ given by

$$
\widehat{\psi}_{k}(\omega)= \begin{cases}(2 \omega)^{\frac{\alpha+1}{2}-k} L_{k}^{(\alpha+1-2 k)}(2 \omega) e^{-\omega}, & \text { for } \omega \geq 0, \\ 0, & \text { for } \omega<0 .\end{cases}
$$

Then

$$
\begin{aligned}
& W^{\alpha}\left(\psi_{k} ; x, y\right)=\frac{1}{2 \pi y^{\frac{\alpha+1}{2}}} \int_{0}^{+\infty} \widehat{\psi}_{k}(y \omega) \widehat{\psi}_{k}(\omega) e^{i \omega x} d \omega \\
& \quad=\frac{2^{\alpha-2 k}}{\pi y^{k}} \int_{0}^{+\infty} \omega^{\alpha+1-2 k} L_{k}^{(\alpha+1-2 k)}(2 \omega) L_{k}^{(\alpha+1-2 k)}(2 y \omega) e^{-\omega(y+1-i x)} d \omega .
\end{aligned}
$$

Denote $p:=\frac{y+1-i x}{2}=\frac{1-i z}{2}$ with $z=x+i y$, one can get

$$
\begin{aligned}
& W^{\alpha}\left(\psi_{k} ; x, y\right)=\frac{1}{4 \pi} y^{-k} \int_{0}^{\infty} \omega^{\alpha+1-2 k} L_{k}^{(\alpha+1-2 k)}(\omega) L_{k}^{(\alpha+1-2 k)}(y \omega) e^{-\omega p} d \omega \\
& =y^{-k} \frac{\Gamma(\alpha+2)}{4 \pi(k !)^{2}} \frac{(p-1)^{k}(p-y)^{k}}{p^{\alpha+2}}{ }_{2} F_{1}\left(-k,-k ;-\alpha-1 ; \frac{p(p-1-y)}{(p-1)(p-y)}\right) \\
& =y^{-k} \frac{\Gamma(\alpha+2)}{4 \pi(k !)^{2}} \frac{(p-1)^{k}(p-y)^{k}}{p^{\alpha+2}}\left(\frac{y}{(p-1)(p-y)}\right)^{k} . \\
& \cdot{ }_{2} F_{1}\left(-k, k-\alpha-1 ;-\alpha-1 ; \frac{p(p-1-y)}{-y}\right) \\
& =\frac{2^{\alpha} \Gamma(\alpha+2)}{\pi(k !)^{2}} \frac{1}{(1-i z)^{\alpha+2}}{ }_{2} F_{1}\left(-k, k-\alpha-1 ;-\alpha-1 ; \frac{|1-i z|^{2}}{4 y}\right),
\end{aligned}
$$

where ${ }_{2} F_{1}(a, b ; c ; t):=\sum_{n=0}^{\infty} \frac{(a)_{n}(b)_{n}}{(c)_{n} n !} t^{n}$ is the hypergeometric function with $(a)_{0}:=$ $1,(a)_{n}:=a(a+1) \cdots(a+n-1)$.

For $z \in U, g^{-1}=\left(\begin{array}{cc}\cos \frac{\theta}{2} & -\sin \frac{\theta}{2} \\ \sin \frac{\theta}{2} & \cos \frac{\theta}{2}\end{array}\right) \in S O(2)$, denote

$$
\omega:=u+i v=g(z)=\frac{z \cos \frac{\theta}{2}-\sin \frac{\theta}{2}}{z \sin \frac{\theta}{2}+\cos \frac{\theta}{2}}
$$




$$
\text { then } \begin{aligned}
& \frac{|1-i \omega|^{2}}{4 v}=\frac{|1-i z|^{2}}{4 y} \text { and } \\
& R_{\theta} W^{\alpha}\left(\psi_{k} ; x, y\right) \\
= & \frac{\left(i \sin \frac{\theta}{2}+\cos \frac{\theta}{2}\right)^{\alpha+2}}{\left(z \sin \frac{\theta}{2}+\cos \frac{\theta}{2}\right)^{\alpha+2}} \frac{2^{\alpha} \Gamma(\alpha+2)}{\pi(k !)^{2}(1-i \omega)^{\alpha+2}}{ }_{2} F_{1}\left(-k, k-\alpha-1 ;-\alpha-1 ; \frac{|1-i \omega|^{2}}{4 v}\right) \\
= & \frac{2^{\alpha} \Gamma(\alpha+2)}{\pi(k !)^{2}(1-i z)^{\alpha+2}}{ }_{2} F_{1}\left(-k, k-\alpha-1 ;-\alpha-1 ; \frac{|1-i z|^{2}}{4 y}\right) \\
= & W^{\alpha}\left(\psi_{k} ; x, y\right) .
\end{aligned}
$$

That is $W^{\alpha}\left(\psi_{k} ; x, y\right)$ is $S O(2)$-invariant. The proof of Theorem 1 is completed.

Remark 1. The differential operator $D_{\nu}$ given by (2.6) is equivalent to the Casimir operator of the representation $U^{\nu}$ of $S L(2, R)$ given by $(2.2)$. In fact, the Casimir operator $\square_{\nu}$ is given by

$$
\square_{\nu}:=-y^{2}\left(\frac{\partial^{2}}{\partial x^{2}}+\frac{\partial^{2}}{\partial y^{2}}\right)+i \nu y\left(\frac{\partial}{\partial x}+i \frac{\partial}{\partial y}\right),
$$

and we have (see [7], [3])

$$
\square_{\nu} W^{\alpha}(\psi, f ; x, y)=\frac{1}{2 \pi y^{\frac{\alpha+1}{2}}} \int_{0}^{\infty}\left(D_{\nu} \widehat{\psi}\right)(y \omega) \widehat{f}(\omega) e^{i \omega x} d \omega .
$$

We shall also note here that function $\psi$ given by (2.3) satisfies (1.4) since $\frac{\alpha+1}{2}-k>$ 0 .

Remark 2. If $\alpha$ is not an integer and $\psi$ is the function defined by (2.3), then for $\theta \in[0,2 \pi], R_{\theta} W^{\alpha}(\psi ; x, y)$ equals to $W^{\alpha}(\psi ; x, y)$ (up to a constant on the unit circle).

\section{ACKNOWLEDGEMENTS}

The author would like to express his thanks to the anonymous referee for many helpful suggestions to this paper.

\section{REFERENCES}

[1] L. Auslander and I. Gertner, Wideband ambiguity functions and the $a \cdot x+b$ group, in "Signal Processing: Part I - Signal Processing Theory", L. Auslander et al. eds., Springer-Verlag, New York, 1990, 1-12. MR 90j:94005

[2] I. Daubechies, "Ten Lectures on Wavelets", SIAM, Philadelphia, 1992. MR 93e:42045

[3] I. Daubechies, J. Klauder and T. Paul, Wiener measures for path integrals with affine kinematic variables, J. Math. Phys., 28(1987), 85-102. MR 88g:81030

[4] D. Gabor, Theory of communication, J. Inst. Electr. Eng., 93(1964), 429-457.

[5] A. Grossmann and J. Morlet, Decomposition of Hardy functions into square integrable wavelets of constant shape, SIAM. J. Math. Anal., 15 (1984), 723-736. MR 85i:81146

[6] C. Heil and D. Walnut, Continuous and discrete wavelet transform, SIAM. Rev., 31(1989), 628-666. MR 91c:42032

[7] Q. Jiang and L. Peng, Casimir operator and wavelet transform, in "Harmonic Analysis in China", Hongkong, Kluwer Academic Publishers, 1995. MR 96m:22016

[8] G. Kaiser, "A Friendly Guide to Wavelet", Birkhäuser, 1994.

[9] E. Kalnins and W. Miller, A note on group contractions and radar ambiguity functions, in "Radar and Sonar, Part II", F. Alberto Grünbaum et al. eds., Springer-Verlag, New York, 1992, 71-82. MR 94g:78006

[10] P. Maas, Wideband approximation and wavelet transform, in "Radar and Sonar, Part II", F. Alberto Grünbaum et al. eds., Springer-Verlag, New York, 1992, 83-88. MR 93m:00045 
[11] W. Magnus, F. Oberhettinger and R. Soni, "Formulas and Theorems for the Special Functions of Mathematical Physics", Springer-Verlag Berlin, Heidelberg, 1966. MR 38:1291

[12] W. Miller, Topics in harmonic analysis with applications to radar and sonar, in "Radar and Sonar, Part I", R. Bluhat et al. eds., Springer-Verlag, New York, 1991, 66-168. MR 93m:00045

[13] P. Morse, Diatomic molecules according to the wave mechanics. II. Vibrational levels, Physical Review, 34(1929), 57-64.

[14] H. Naparst, Dense target signal processing, IEEE Trans. Inform. Theory, 37(1991), 317-327. MR 92b:94009

[15] W. Schempp, "Harmonic Analysis on the Heisenberg Nilpotent Lie Group, with Applications to Signal Theory", Longman, 1986. MR 88f:22025

[16] D. Swick, An ambiguity function independent of assumption about bandwidth and carrier frequency, NRL Report 6471, Washington, DC, 1966.

[17] D. Swick, A review of wide-band ambiguity function, NRL Report 6994, Washington, DC, 1969.

[18] L. Weiss, Wavelets and wideband correlation processing, IEEE Signal Proc. Magazine, Jan. 1994, 13-32.

[19] C. Wilcox, The synthesis problem for radar ambiguity functions, in "Radar and Sonar, Part I", R. Bluhat et al. eds., Springer-Verlag, New York, 1991, 229-260. MR 93m:00045

[20] P. Woodward, "Probability and Information Theory with Applications to Radar", 2nd ed, Pergamon Press, New York, 1964. MR 31:4637

[21] R. Young, "Wavelet Theory and Its Applications", Kluwer Academic Publishers, Boston, 1993. MR 95j:94007

Department of Mathematics, Peking University, Beijing 100871, People's Republic of CHINA

Current address: Department of Mathematics, National University of Singapore, Lower Kent Ridge Road, Singapore 119260

E-mail address: qjiang@haar.math.nus.sg 